This item was submitted to Loughborough's Research Repository by the author.

Items in Figshare are protected by copyright, with all rights reserved, unless otherwise indicated.

\title{
Simulation of inertial behavior of dilute particle flow in horizontal channel with Eulerian method of velocity-reassociated quadrature-based method of moments
}

\section{PLEASE CITE THE PUBLISHED VERSION}

http://dx.doi.org/10.1016/j.powtec.2016.09.035

\section{PUBLISHER}

(C) Elsevier

\section{VERSION}

AM (Accepted Manuscript)

\section{PUBLISHER STATEMENT}

This work is made available according to the conditions of the Creative Commons Attribution-NonCommercialNoDerivatives 4.0 International (CC BY-NC-ND 4.0) licence. Full details of this licence are available at: https://creativecommons.org/licenses/by-nc-nd/4.0/

\section{LICENCE}

CC BY-NC-ND 4.0

\section{REPOSITORY RECORD}

Sun, Dan, Andrew Garmory, and Gary Page. 2016. "Simulation of Inertial Behavior of Dilute Particle Flow in Horizontal Channel with Eulerian Method of Velocity-reassociated Quadrature-based Method of Moments". figshare. https://hdl.handle.net/2134/23311. 


\title{
Simulation of inertial behavior of dilute particle flow in horizontal pipe with Eulerian method of Velocity-reconstructed Quadrature-based Method of
}

\section{Moments}

Dan Sun, Andrew Garmory, Gary J. Page

Department of Aeronautical and Automotive Engineering,

Loughborough University, Loughborough, UK

dansuncfd@outlook.com

\begin{abstract}
The velocity reconstructed two-node quadrature-based method of moments (VR-QMOM) is a robust Eulerian method which is able to predict the crossing trajectory and surface bouncing phenomena for dilute particle flow. It is used here to predict the particle-laden flow in the dilute particle concentration in a horizontal channel. The multiphase simulation was carried out in the Eulerian-Eulerian two-phase model with VR-QMOM for the particle phase and for comparison with the more established Eulerian-Lagrangian two-phase flow method. The gas phase is simulated with RANS equations and discretised with second-order upwind spatial numerical schemes. Comparing the predicted particle flows obtained using the VR-QMOM and Eulerian-Lagrangian approaches, the conclusion can be made that the VR-QMOM can successfully predict bouncing multiphase flows with dilute particle phase. Both trajectory crossing and bouncing behaviour and the trend with increasing particle size are reproduced. The two-node VR-QMOM is seen to be an alternative methodology for the prediction of dispersed particle flow problems.
\end{abstract}

Keywords: Multiphase flow; Eulerian method; quadrature method of moment; VR-QMOM; horizontal pipe 


\section{Introduction}

Pneumatic conveying is widely used for granular transportation in industries from chemical, food and pharmaceutical processing to power generation and metallurgical engineering. [1, 2, 3,4] The conveying systems are capable of transporting almost any kind of material. In practice, the conveying distance of the single stage of the system can reach $1.5 \mathrm{~km}$ for most applications. [5]. For the dilute phase conveying, the minimum value of the conveying air velocity is approximately 13 $15 \mathrm{~m} / \mathrm{s}$. The particles are suspended in the dilute phase conveying system and if the air velocity is lower than the minimum value required for the dilute phase conveying, then saltation in the horizontal pipeline and chocking in the vertical riser may occur. Some materials can be conveyed at lower velocity as non-suspension particle flows in the dense phase flow mode; for example as in the moving bed and the plug flow mode which are used for short distance conveying.

There are three general categories for models used in pneumatic conveying process and fluidized beds for dense flow: fluidized dense phase, plug flow and dilute phase only [6], Here we are interested in the particle laden flow in the dilute phase conveying system, where the flow behaviours of particles are controlled by the gas-particle interphase exchange, the particle-wall collisions and the collisional interactions of particles.

Numerous experimental studies have been carried out for particle laden flow in horizontal channels looking at the particle flow behaviour covering the effects of particle size, mass loading ratio, channel height, wall roughness and gas Reynolds numbers. [7, 8, 9,10,11,12,13]. From experiments, particle bouncing and particle trajectory crossing have been observed [10], which are characterized as typical flow phenomena for a dilute particle concentration. Experimental data mentioned above also indicated that in horizontal pipes the particle inertia behaviour effect on particle motion caused veering off the flow path of the carrier fluid path significantly, and the gravity and surface confinement which result in a subsequent collision with walls. 
In the numerical simulation of the solid flow, Lagrangian methods are used to trace the trajectories of the solid particles directly and accurately. Tsuji et al. $[14,15,16]$ used Lagrangian simulation to predict the continuous bounce of particles in a horizontal pipe under the influence of gravity with an abnormal bouncing model and the three-dimensional collision model for a particle of arbitrary shape with a flat wall. Sommerfeld et al. [17,18] used a Lagrangian method to analyse the collision between spherical solid particles in a horizontal channel with a rough wall as well as inter-particle collisions. Squires et al. [19] studied the effect of wall roughness on dispersed phase properties of particle laden turbulent channel flow using discrete particle simulation for the particulate phase. The motion of particles was dictated by their interactions with the local turbulent fluid and wall collision. Li et al. [20] studied the effects of friction and restitution coefficients of particles on horizontal pneumatic conveying by the discrete element method of particles. Recently, new numerical methods are used to predict the dispersed particle flow. For example, the stochastic direct simulation Monte Carlo algorithm was used to predict the particle trajectories of dilute particle flow by Pawar et al [21], whilst Zhou et al. also used an immersed boundary lattice Boltzmann method to study the particle-laden flows. [22]

These particle trajectories of the particle-laden flow observed in both experiments and numerical studies show the two typical characteristics of the particle flow: trajectory crossing and wall bouncing - which give the evidence that the particle-particle collisions are less important in the dilute particle regime. The particles are individually suspended in the flow field, and the particles are dominated by the particle inertia and interaction between the fluid phase and the particles. Therefore, particle-particle collision is neglected at this stage of the current work.

The basic Eulerian method has, in the past, failed to capture the particle trajectories and wall bouncing phenomena in the dilute phase with the mean velocity field. The ill-posedness of the Eulerian method was investigated by predicting the particle trajectory crossing flow of inertial particle transportation. [23] However, the velocity-based quadrature-based method of moment 
(QMOM) proposed by Fox [24] offered an Eulerian method to predict particle disperse flows with multiple velocity fields. This quadrature-based third-order moment method (QMOM) models the kinetic momentum transfer of the particles based on the kinetic theory of gases [25], associated with the Gaussian quadrature method [26] by approximating the moments of velocity distribution, $\mathrm{M}_{\mathrm{kl} \cdots \mathrm{w}}=\sum_{\alpha=1}^{\mathrm{n}} \mathrm{n}_{\alpha} v_{\alpha k} v_{\alpha l} \cdots v_{\alpha w}$. It is capable of predicting crossing trajectories and bouncing particles, however, the set of 20 moments and 8 weighted velocity fields has a relatively high computational overhead. Desjardins et al [27] proposed a simpler two-node QMOM method for dilute fluid-particle flows and overcame the deficiency of the Eulerian method with a more affordable approach using only 8 moments and 2 velocity fields. The two velocity fields are represented by the two node sequences $\left[n_{1}, v_{1, \mathrm{i}}, v_{1, \mathrm{j}}, v_{1, \mathrm{k}}\right]$ and $\left[n_{2}, v_{2, i}, v_{2, j}, v_{2, k}\right]$. Unfortunately, this two-node QMOM failed to predict some trajectory-crossing problems under certain circumstances (this will be shown and discussed in detail in section 3).

In this work, a new robust Eulerian model is used to model dilute particles in the horizontal conveying channel. An alternative velocity reconstructed two-node QMOM is proposed with a new two-node closure approach with thirteen moments for the product-difference algorithm to reconstruct the node sequence of the two particle flow fields. The crossing trajectory and wall bouncing behaviour by two particle jets are predicted with this velocity reconstructed quadrature method of moment (VR-QMOM) in a horizontal channel.

\section{Gas-solid two-phase model with VR-QMOM for particles}

The dynamics of the multiphase flow in the horizontal channel is simulated by the Eulerian Lagrangian and Eulerian-Eulerian two-phase framework. The continuous gas phase is modelled by the Reynolds Averaged Navier-Stokes equations with the RNG $k-\varepsilon$ turbulence model and standard wall functions. The numerical scheme for the gas phase is the second order quadratic upwind method. The particulate phase is calculated by the velocity reconstructed two-node quadraturebased method of moment (VR-QMOM) and, for comparison, with the Lagrangian method using the 
commercial CFD software ANSYS Fluent. The VR-QMOM is implemented in structured grid research CFD code.

In this simulation the particle concentration is of the dilute regime. One-way gas-particle coupling is assumed and the inter-particle collisions are neglected. The particles are currently considered as spherical and monodispersed. The ideally smooth surface of the wall and the particles is studied to show the capability of the Eulerian method in predicting trajectory crossing and wall bouncing of particles in the dilute concentration.

\subsection{Moment transportation equations}

The principle of the VR-QMOM model is based on the method of moment closed with the two node velocity reconstructed quadrature algorithm. in terms of the method of moment, the transport equations are established by the kinetic theory of gases [28],

$$
\frac{\partial f}{\partial t}+\mathbf{U} \cdot \frac{\partial f}{\partial \mathbf{x}}+\frac{\partial f}{\partial U}\left(f \frac{\mathbf{F}}{m_{p}}\right)=\mathbb{C}
$$

where $f$ is the velocity distribution function, $\mathbf{U}$ is the particle velocity vector, and $m_{p}$ is the mass of a single particle. $\mathbb{C}$ is the particle inter-collision term, where $\mathbb{C}=0$ for dilute particle flow. $\mathbf{F}$ in the last term on LHS is the external force, including gravity and the drag force. This combined force can be expressed as

$$
\mathbf{F}\left(\mathbf{U}_{\mathbf{f}}, \mathbf{U}\right)=\frac{m_{p}}{\tau_{p}}\left(\mathbf{U}_{\mathbf{f}}-\mathbf{U}\right)+m_{p} \mathbf{g}
$$

where $\tau_{p}$ is the characteristic time for the relaxation of particle velocity to the fluid velocity, determined as,

$$
\frac{1}{\tau_{p}}=\frac{3 \rho_{f}}{4 d_{p} \rho_{p}} C_{D}\left|\mathbf{U}_{\mathbf{f}}-\mathbf{U}\right|
$$

where $\rho_{f}$ is the fluid density, $\rho_{p}$ is the particle density, $d_{p}$ is the particle diameter, and $\mathbf{U}_{\mathbf{f}}$ is the fluid velocity vector. $C_{D}$ is the particle drag coefficient given by [29],

$$
C_{D}=\frac{24}{R e_{p}}\left(1+0.15 R e_{P}^{0.687}\right)
$$

and $R e_{P}=\rho_{f} d_{p}\left|\mathbf{U}_{\mathbf{f}}-\mathbf{U}\right| / \mu_{f}$ is particle Reynolds number, where $\mu_{f}$ is the kinematic viscosity of the fluid phase. 
The two-node quadrature approach uses number density $\left(n_{1}, n_{2}\right)$ and particle velocities $\left(\mathbf{U}_{\mathbf{1}}, \mathbf{U}_{\mathbf{2}}\right)$ to represent the particle flow field. The velocity distribution of dispersed particles can be expressed by the delta function $f=n_{1} \delta\left(\mathbf{U}-\mathbf{U}_{1}\right)+n_{2} \delta\left(\mathbf{U}-\mathbf{U}_{2}\right)$. The $s$ order moment of particle velocities in the QMOM model is defined by,

$$
M_{i j \ldots l}^{s}=n_{1} U_{1 i} U_{1 j} \cdots U_{1 l}+n_{2} U_{2 i} U_{2 j} \cdots U_{2 l}
$$

Then the moment transport equations for particles can finally be obtained from Eq (1) as,

$$
\begin{aligned}
& \frac{\partial M^{0}}{\partial t}+\frac{\partial M_{k}^{1}}{\partial x_{k}}=0 \\
& \frac{\partial M_{i}^{1}}{\partial t}+\frac{\partial M_{i k}^{2}}{\partial x_{k}}=\frac{n_{1}}{m_{p}} F_{1, i}+\frac{n_{2}}{m_{p}} F_{2, i} \\
& \frac{\partial M_{i j}^{2}}{\partial t}+\frac{\partial M_{i j k}^{3}}{\partial x_{k}}=\frac{n_{1}}{m_{p}} U_{1, i} F_{1, j}+\frac{n_{1}}{m_{p}} U_{1, j} F_{1, i}+\frac{n_{2}}{m_{p}} U_{2, i} F_{2, j}+\frac{n_{2}}{m_{p}} U_{2, j} F_{2, i} \\
& \frac{\partial \mathrm{M}_{\mathrm{iii}}^{3}}{\partial \mathrm{t}}+\frac{\partial \mathrm{M}_{\mathrm{iik}}^{4}}{\partial \mathrm{x}_{\mathrm{k}}}=3 \frac{\mathrm{n}_{1}}{\mathrm{~m}_{\mathrm{p}}}\left(\mathrm{U}_{1, \mathrm{i}}\right)^{2} \mathrm{~F}_{1, \mathrm{i}}+3 \frac{\mathrm{n}_{2}}{\mathrm{~m}_{\mathrm{p}}}\left(\mathrm{U}_{2, \mathrm{i}}\right)^{2} \mathrm{~F}_{2, \mathrm{i}}
\end{aligned}
$$

The terms on the right hand side represent interactions due to the influence from gravity $\mathbf{g}$ and interactions with fluid phase.

In VR-QMOM, the moment equations (6) are closed by 13 moment transportations in a 3 dimensional system,

$$
W^{3}=\left(M^{0}, M_{i}^{1}, M_{j}^{1}, M_{k}^{1}, M_{i i}^{2}, M_{j j}^{2}, M_{k k}^{2}, M_{i j}^{2}, M_{j k}^{2}, M_{i k}^{2}, M_{i i i}^{3}, M_{j j j}^{3}, M_{k k k}^{3}\right) \in \mathbb{R}^{13} .
$$

where the moments of particles in this 13-moment system are expressed as,

$$
\begin{aligned}
& M^{0}=\mathrm{n}_{1}+\mathrm{n}_{2} \\
& M_{i}^{1}=\mathrm{n}_{1} \mathrm{U}_{1 \mathrm{i}}+\mathrm{n}_{2} \mathrm{U}_{2 \mathrm{i}} \\
& M_{i j}^{2}=n_{1} U_{1 i} U_{1 j}+n_{2} U_{2 i} U_{2 j} \\
& M_{i i i}^{3}=\mathrm{n}_{1} \mathrm{U}_{1 \mathrm{i}} \mathrm{U}_{1 \mathrm{j}} \mathrm{U}_{1 \mathrm{k}}+\mathrm{n}_{2} \mathrm{U}_{2 \mathrm{i}} \mathrm{U}_{2 \mathrm{j}} \mathrm{U}_{2 \mathrm{k}}
\end{aligned}
$$

\subsection{Two-node quadrature-based closure approach with thirteen moments}

The key for the VR-QMOM model is to enclose the QMOM using the quadrature approach to find the velocity fields, number densities and velocities of the particles, with the updated moments 
from the moment equations. This closure approach of VR-QMOM includes two steps. The first step is the calculation of number densities and velocities with the product-difference (PD) algorithm, and the second step is to associate the number densities and velocities using the cross moments to set up the node sequences for the two flow fields.

The mean velocity is defined by $U_{p i}=M_{i}^{1} / M^{0}$, and then the velocity deviation is

$$
a_{1,2}=\mathbf{U}_{1,2}-\mathbf{U}_{\mathbf{p}}
$$

Accordingly, the two-node Gaussian quadrature-based moment of velocity deviation of each node is defined as

$$
a_{i j \cdots l}=n_{1} c_{1 i} c_{1 j} \cdots c_{1 l}+n_{2} c_{2 i} c_{2 j} \cdots c_{2 l}
$$

The first three order moments of velocity deviation $\mathbf{c}$ are expressed as

$$
\begin{aligned}
& a_{0}=n_{1}+n_{2} \\
& a_{\mathrm{i}}=\mathrm{n}_{1} \mathrm{c}_{1 \mathrm{i}}+\mathrm{n}_{2} \mathrm{c}_{2 \mathrm{i}} \\
& a_{i j}=n_{1} c_{1 i} c_{1 j}+n_{2} c_{2 i} c_{2 j} \\
& a_{i i i}=n_{1} c_{1 i}^{3}+n_{2} c_{2 i}{ }^{3}
\end{aligned}
$$

The moments of velocity deviation in equation (11) can be calculated straightforwardly by the 13-moment system (7). The covariance matrix and the third-order velocity variance can be represented by,

$$
\begin{aligned}
{\left[a_{i j}\right] } & =\left[\begin{array}{lll}
M_{i i}^{2} / M^{0}-U_{p i} U_{p i} & M_{i j}^{2} / M^{0}-U_{p i} U_{p j} & M_{i k}^{2} / M^{0}-U_{p i} U_{p k} \\
M_{i j}^{2} / M^{0}-U_{p i} U_{p j} & M_{j j}^{2} / M^{0}-U_{p j} U_{p j} & M_{j k}^{2} / M^{0}-U_{p j} U_{p k} \\
M_{i k}^{2} / M^{0}-U_{p i} U_{p k} & M_{j k}^{2} / M^{0}-U_{p j} U_{p k} & M_{k k}^{2} / M^{0}-U_{p k} U_{p k}
\end{array}\right] \\
{\left[a_{i i i}\right] } & =\left[\begin{array}{l}
\frac{1}{M^{0}} M_{i i i}^{3}-\left(U_{p i}\right)^{3}-3 a_{i i} U_{p i} \\
\frac{1}{M^{0}} M_{j j j}^{3}-\left(U_{p j}\right)^{3}-3 a_{j j} U_{p j} \\
\frac{1}{M^{0}} M_{k k k}^{3}-\left(U_{p k}\right)^{3}-3 a_{k k} U_{p k}
\end{array}\right] .
\end{aligned}
$$

The two-node quadrature closure by McGraw [30] is used to calculate the weights and abscissas for each Cartesian direction. 


$$
\begin{aligned}
& c_{1 \mathrm{i}}=-\left(\varepsilon_{\mathrm{i}} \sqrt{\mathrm{a}_{\mathrm{ii}}}\right) \sqrt{\frac{n_{2}}{n_{1}}} \\
& c_{2 i}=\left(\varepsilon_{i} \sqrt{a_{i i}}\right) \sqrt{\frac{n_{1}}{n_{2}}}
\end{aligned}
$$

Where we define variance $x$ as the deviation of weights,

$$
n_{1,2}=\left(\frac{1}{2} \pm x\right) M^{0}
$$

In the two-node closure approach, the weight variance $x$ is identified for all component of particle velocities.

$$
x=\frac{a_{i i i}}{2 \sqrt{\left(a_{i i i}\right)^{2}+4\left(a_{i i}\right)^{3}}}, \quad a_{i i} \neq 0, i=i, j, k
$$

The $\varepsilon_{i}$ in equation (14) represents the correlation between the abscissas and the weights and $\left|\varepsilon_{i}\right|=1$.

The second step for the two-node quadrature-based thirteen-moment closure is to associate the weights and abscissas by calculating the $\operatorname{sign} \varepsilon_{i}$. From Eq. (11),

$$
\varepsilon_{j}=\operatorname{sign}\left(a_{i j}\right) / \varepsilon_{i}
$$

assuming that in the direction of $i, \varepsilon_{i}=1$, the particle velocity can be calculated by

$$
\begin{aligned}
& \mathrm{U}_{1 \mathrm{i}}=\mathrm{U}_{\mathrm{pi}}-\frac{a_{i j}}{\sqrt{a_{i i}}} \sqrt{\frac{n_{2}}{n_{1}}} \\
& \mathrm{U}_{2 \mathrm{i}}=\mathrm{U}_{\mathrm{pi}}+\frac{a_{i j}}{\sqrt{a_{i i}}} \sqrt{\frac{n_{1}}{n_{2}}}
\end{aligned}
$$

A unique pair of weighted velocity fields is obtained from the set of moments. Thus, the velocities of the two nodes are reconstructed by the second-order cross-moments. The robust Eulerian method of VR-QMOM is defined for dilute free particle flow.

\subsection{Boundary conditions}

To give the desired surface bouncing behaviour, it is necessary to specify appropriate boundary conditions for the dispersed phase. Here we achieve this by using 'ghost cells' at the edge of the domain for which the two weighted velocity fields are found from the values in the first real cell according to 


$$
\left[\begin{array}{c}
n_{\alpha} \\
U_{\alpha i} \\
U_{\alpha j} \\
U_{\alpha k}
\end{array}\right]_{g c}=\left[\begin{array}{c}
n_{\alpha} / e_{T} \\
e_{i} U_{\alpha i} \\
e_{j} U_{\alpha j} \\
e_{k} U_{\alpha k}
\end{array}\right\rceil_{1}
$$

where $e_{i}, e_{j}$ and $e_{k}$ are the coefficients of restitution in the three directions between the particle and wall. They are specified separately here for completeness in cases, such as those involving erosion, where the normal and tangential restitution coefficients are important. The total velocity coefficient of restitution $e_{T}$ is defined as

$$
e_{T}=\frac{\sqrt{\left(e_{i} U_{i}\right)^{2}+\left(e_{j} U_{j}\right)^{2}+\left(e_{k} U_{k}\right)^{2}}}{\sqrt{U_{i}^{2}+U_{j}^{2}+U_{k}^{2}}}
$$

In the case of perfectly elastic collisions of a surface normal to the $j$-direction note that $e_{i}=$ $1, e_{j}=-1$ and $e_{k}=1$.

\subsection{Numerical Schemes}

The QMOM requires the moments to be updated using the weighted velocity nodes. The transport equation for the general moment $\mathrm{M}^{3}{ }_{\mathrm{abc}}$ can be written as

$$
\frac{\partial M_{a b c}^{3}}{\partial t}+\frac{\partial n_{1} U_{1 a} U_{1 b} U_{1 c} U_{1 i}}{\partial x_{i}}+\frac{\partial n_{2} U_{2 a} U_{2 b} U_{2 c} U_{2 i}}{\partial x_{i}}=S
$$

where $S$ represents source terms such as those due to drag or gravity.

As with any finite volume transport scheme the remaining stage is to find the face fluxes using an appropriate discretisation scheme. It would be possible to apply the discretisation scheme to the moments to find the set of face moments and then convert them to the velocities, but following [31] we apply the discretisation scheme to the velocity fields as if high-order schemes are used to discretise the moments. Thus, the non-realizable combinations of moments can be produced. The second-order transport TVD scheme is used here

$$
\begin{aligned}
& \phi_{\alpha, f}=\phi_{\alpha, U P}+\frac{1}{2} \psi(\rho)\left(\phi_{\alpha, D O W N}-\phi_{\alpha, U P}\right) \\
& \rho=\frac{\phi_{\alpha, U P 2}-\phi_{\alpha, U P}}{\phi_{\alpha, D O W N}-\phi_{\alpha, U P}}
\end{aligned}
$$


where, if $\phi_{\alpha, f}$ represents any velocity component of node $\alpha$ on face $f$. 'UP2' represents the cell upwind of the upwind cell. The limiter function $\psi(\rho)$ needs to be specified and we do that here using Minmod. [32]

$$
\psi(\rho)=\frac{1}{2} \max (0, \min (\rho, 1))
$$

\section{Robustness of the VR-QMOM model}

Comparing with the original two-node eight-moment QMOM model [27], the new two-node thirteen-moment VR-QMOM model uses the extra moment to establish the relationship between the three components of velocity of each node. Whilst in the original two-node eight-moment QMOM model, it assumes that $\varepsilon_{i}=\varepsilon_{j}=\varepsilon_{k}=1$ when converting moment to velocity. Figure 1 shows how this leads to the failed prediction of the crossing trajectory of two particle streams injecting from both bottom and left boundaries in the 2-D simulation. In the lower concentration of particles, the two flow stream of particles should cross at the centre of the flow domain as shown Fig1-a simulated by the Lagrangian method. However, the simulation of the particle crossing with the twonode eight-moment model gives an incorrect prediction caused by the assumption mentioned above. If the velocities of the two nodes in the flow field are $\left[\mathrm{u}_{1}, \mathrm{v}_{1}\right]=[0,1]$ and $\left[\mathrm{u}_{2}, \mathrm{v}_{2}\right]=[1,0]$, then this assumption, $\varepsilon_{i}=\varepsilon_{j}=1$, will force the velocities of the crossed node calculated from the equations $(8,13)$ to be $\left[\mathrm{u}_{1, \mathrm{c}}, \mathrm{v}_{1, \mathrm{c}}\right]=[0.5,0.5]$ and $\left[\mathrm{u}_{2, \mathrm{c}}, \mathrm{v}_{2, \mathrm{c}}\right]=[0.5,0.5]$, as shown in Fig1-(b).

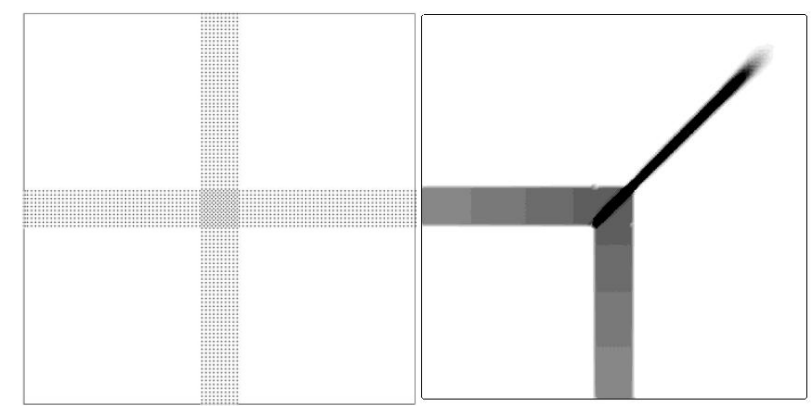

(a) Lagrangian method

(b) two-node QMOM

Figure 1. Particle trajectory and contours of particle number density for two dilute jets with two node eight moment model. 
Using the VR-QMOM model, the predictions of arbitrary crossing trajectories are validated in Figure 2. In the simulation, the two particle flow streams are taken as two separated flow fields. With the extra cross moment of second order moment of velocity, the relationships of the two components of particles velocity are associated correctly. The two particle crossing streams can be assembled as the two particle jets of injecting and rebounded particle streams. Thus, the particlesurface bouncing flow at any impingement angle is correctly predicted using the new VR-QMOM method.
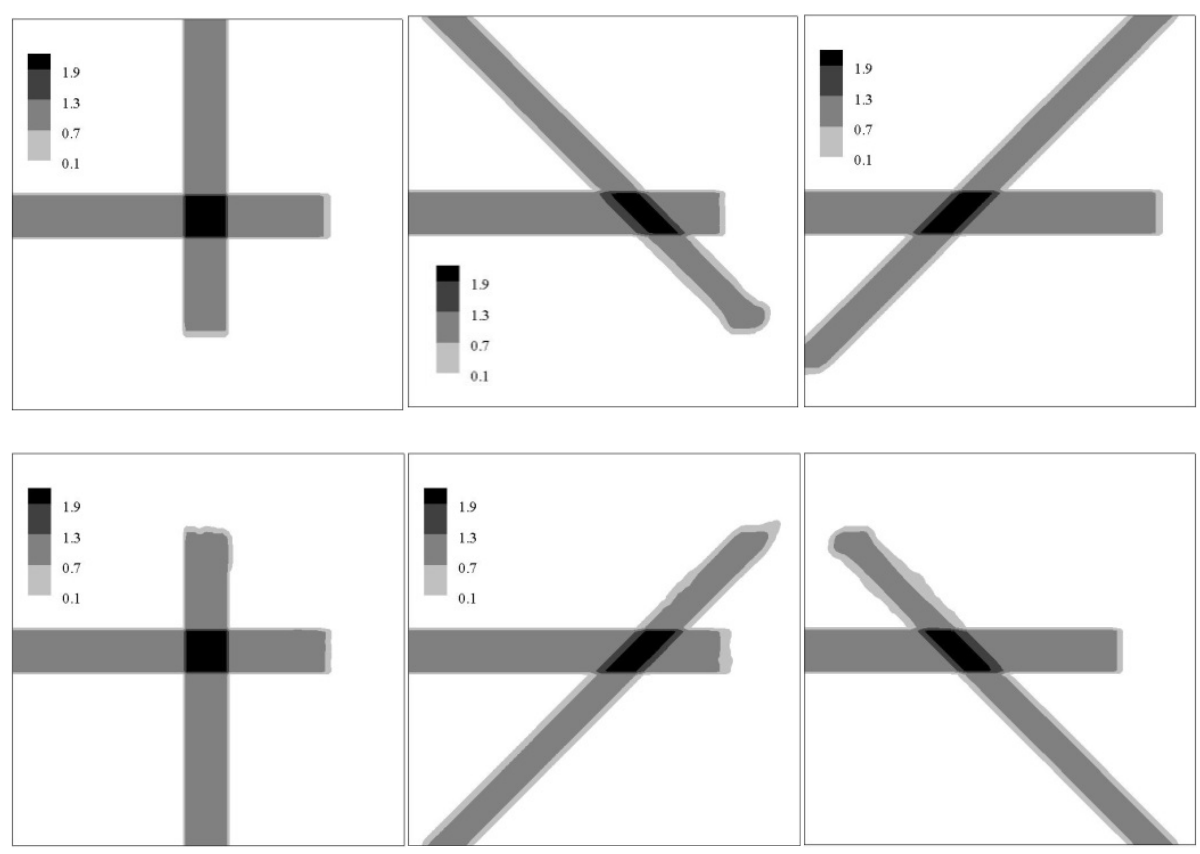

Figure 2. Contours of particle number density for two dilute jets at arbitrary crossing angles with two node thirteen moment model.

\section{Simulation results}

In this section, the two-fluid method with VR-QMOM is used to simulate dilute fluid-particle flows in a horizontal channel to predict the general crossing-trajectory and wall-rebounding particle flows in an Eulerian-Eulerian framework. In the simulation, the horizontal pipe is $35 \mathrm{~mm}$ in height and $6 \mathrm{~m}$ in length [33]. The averaged velocity of gas phase is $20 \mathrm{~m} / \mathrm{s}$. The gas density and dynamic viscosity are $1.25 \mathrm{~kg} / \mathrm{m}^{3}$ and $1.8 \times 10^{-6} \mathrm{Ns} / \mathrm{m}^{2}$ respectively. The density of particles is $2.45 \mathrm{~g} / \mathrm{cm}^{3}$, and the diameters of particles are uniform in each calculation case. 


\subsection{Gas velocity}

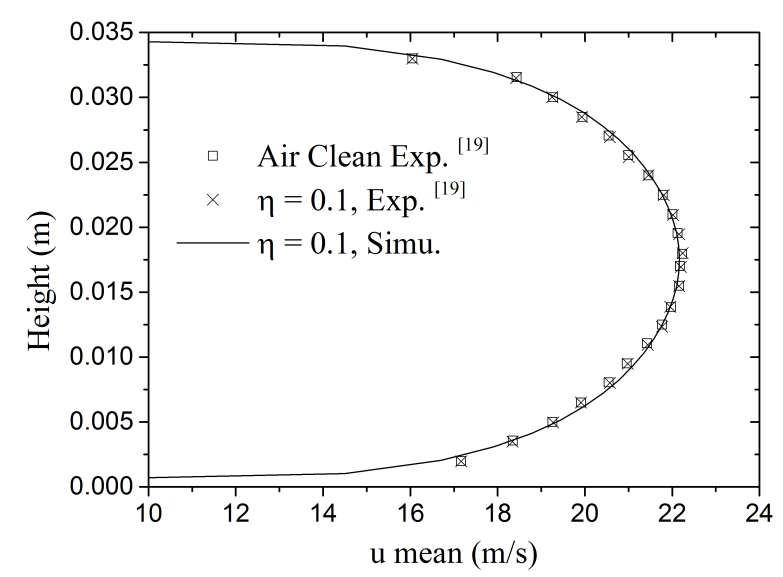

Figure 3. Calculated particle velocities compared with the experiment results [33].

Fig 3 shows the gas velocities at the $5.8 \mathrm{~m}$ distance from the inlet in the horizontal channel. The experimental result is from ref [37], which includes the gas velocities of the clean air injection and the gas velocities with the solid particles at a mass loading ratio $\eta=0.1$. From this figure, the effect of the particle injection with $\eta=0.1$ can hardly observed from the experiment results. Therefore, in this research, the simulated mass load ratio of particles is chosen as $\eta=0.1$, and the two-way gasparticle coupling is neglected. Also, the simulated gas velocity agrees with the experiment as shown in Figure 3. This is the Eulerian-Lagrangian simulation result using the RNG k- $\varepsilon$ turbulence model in the Fluent software. In the Eulerian-Eulerian modelling with VR-QMOM, we retain the same gas flow field from Fluent to ensure the gas flow field is consistent when comparing the two simulation methods.

\subsection{Time evolution}

Figure 4 shows the particle flow in the horizontal channel for a simulated particle diameter of $300 \mu \mathrm{m}$; the particle concentration with VR-QMOM time evolution varies from 0.05 s of the top figure to $0.35 \mathrm{~s}$ at the bottom. The particle flow is controlled mainly by the gas phase carrier and inertial behavior of particles. Affected by the gravity force, the particles hit the surface of the 
bottom wall. At the inlet of the channel, all the particles are injected with the same velocity and same number density. At time $\mathrm{t}=0.05 \mathrm{~s}$, the particle trajectory at the front shows that the particle bounced back after colliding with the bottom surface. As the time evolves, the particles bounce off the top wall driven by the particle inertia, and then fell down to the bottom due to gravity. Then particle rebounding flow are observed in the prediction of particle concentration in the horizontal channel. From the simulation, using the new VR-QMOM method, the particle rebounding flow in the horizontal pipe is successfully predicted, which cannot be achieved by the basic Eulerian method.
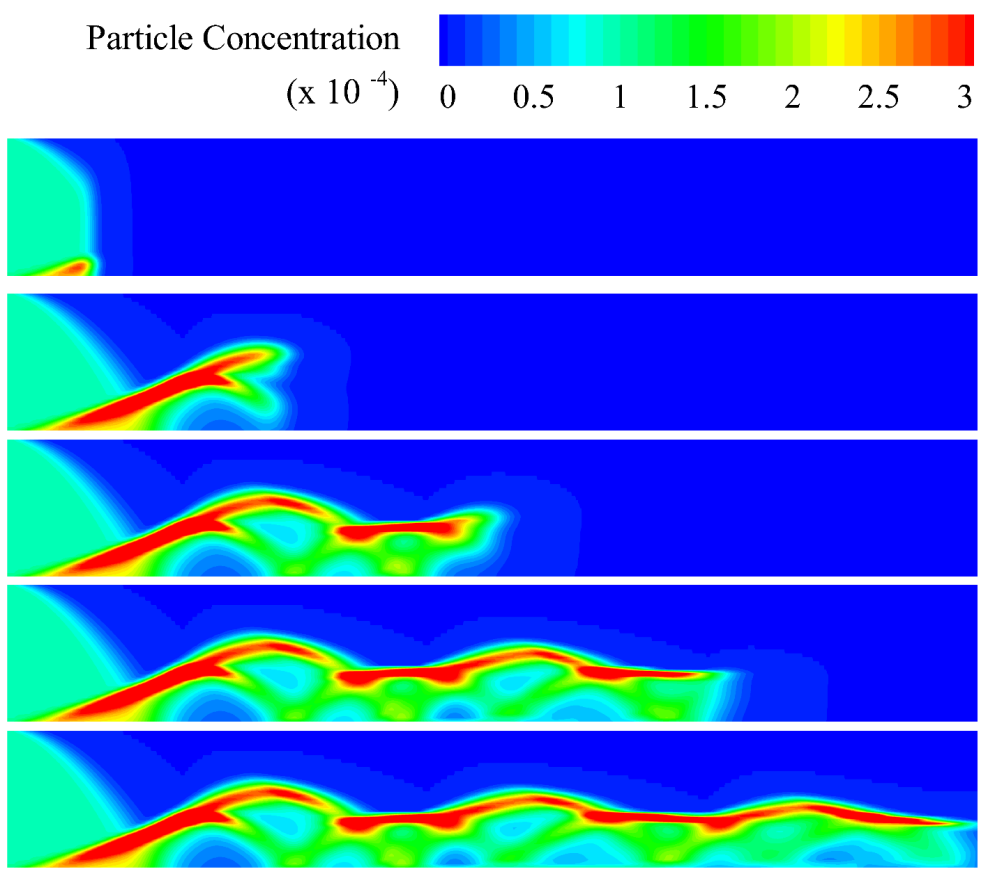

Figure 4. Time evolution of particle concentration in horizontal channel. (from top to bottom $0.05 \mathrm{~s}$, $0.15 \mathrm{~s}, 0.25 \mathrm{~s}, 0.35 \mathrm{~s})$

\subsection{Particle diameter}

The competition of the effects of particle flow with the gas carrier and inertial behavior can be evaluated by the Stokes number $(\mathrm{St})$, which is defined as the ratio of the characteristic time of particle and the characteristic time of flow. If $\mathrm{St}<<1$, the particle flow follows the continuous phase, while for $\mathrm{St}>>1$ the particle flow follows a ballistic trajectory. 
In Figure 5, the effects of the Stokes number are studied by using various diameters of particles. In the left column of the pictures, the particle trajectories are calculated using the Lagrangian method and the trajectories are colored by the particle concentration. It is observed that small particles, such as particles with $30 \mu \mathrm{m}$ diameter, the flow is dominated by drag and the particle trajectories follow the flow path of the gas phase in both Lagrangian and Eulerian simulations; however, for large particle flows these are dominated by inertia. From the simulation, when the particle size increases, the particle bounces higher in the channel, and the inertial behavior of particles is more obvious.

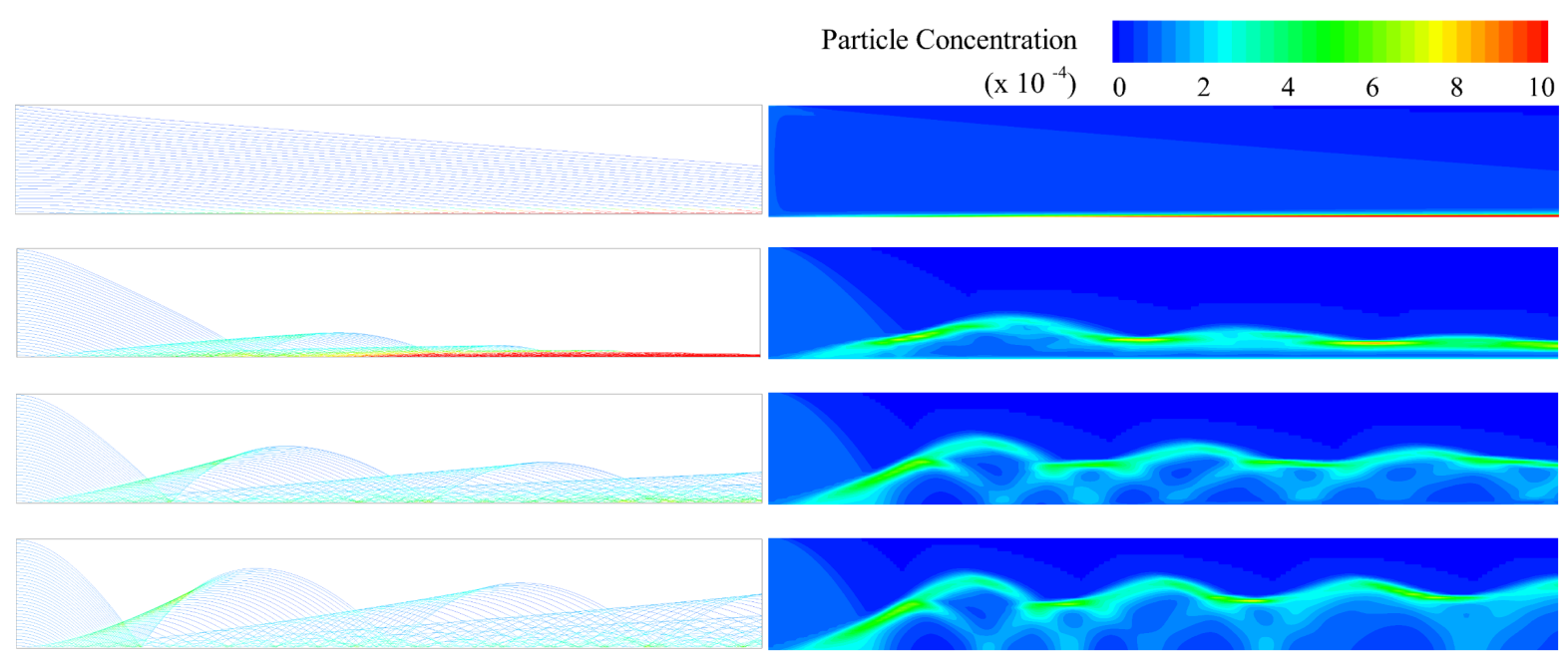

Figure 5. Particle distribution of multiphase flow in horizontal channel (on the left is the calculated particle trajectories with Lagrangian method, on the right is the number densities predicted by VRQMOM; the particle diameters are $30 \mu \mathrm{m}, 130 \mu \mathrm{m}, 300 \mu \mathrm{m}, 625 \mu \mathrm{m}$ from top to bottom)

From the simulations by the new VR-QMOM method, we see that the particle bouncing behavior is accurately modelled when compared with the Lagrangian method plotted on the left. In the distribution of particles of $30 \mu \mathrm{m}$ diameter, the particles followed the flow path of the gas phase, and the particle deposition caused by gravity is observed on the bottom surface. Particles with a diameter of $300 \mu \mathrm{m}$ rebound in the channel. As the particles go forward, the height reduction of particle bounce is affected by the gas phase which drives the particles toward the exit of the channel 
(note the collision of the particle with the wall surface is assumed as elastic). In the distribution of larger particles, such as $625 \mu \mathrm{m}$ particles, the inertia behavior increases the particle bouncing height and then enhances the residence time of particles. Therefore, the VR-QMOM offers an accurate Eulerian method for the dilute particle flow and establishs the Eulerian-Eulerian two-fluid model for the multiphase systems in this research.

\subsection{Particle velocity}

The principle that the Eulerian method VR-QMOM is able to simulate the dilute particle flow is that there are two node of particles which separate the particle flow in two flow fields. At the surface, the particle-wall collision can be predicted by the flow field of the impacting particles and the flow field of reflected particles. In figure 6 , the velocity field of particles are used to illustrate the relationship of the two particle nodes. From the simulation result of the $300 \mu \mathrm{m}$ particle, the detailed particle and velocity distributions are scaled up near the inlet of the horizontal pipe where the particle streams bounce off the surface after the first wave. On the top, the figure shows the particle concentration of the local position. $M^{0}=n_{1}+n_{2}$. In the middle, the particle concentration of the particles in node 1 is plotted at the same position as the top figure, and also the particle velocity vectors of node 1 in this region are plotted as a uniform arrow. The bottom figure shows the particle concentration and the velocity vectors of particles of node 2 .

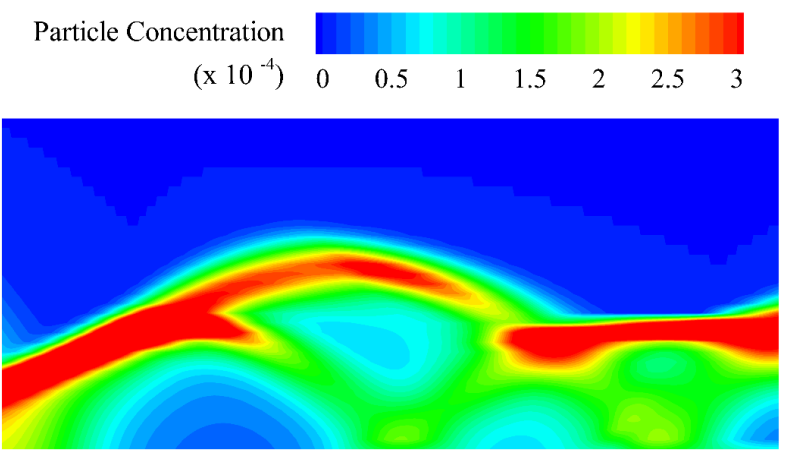



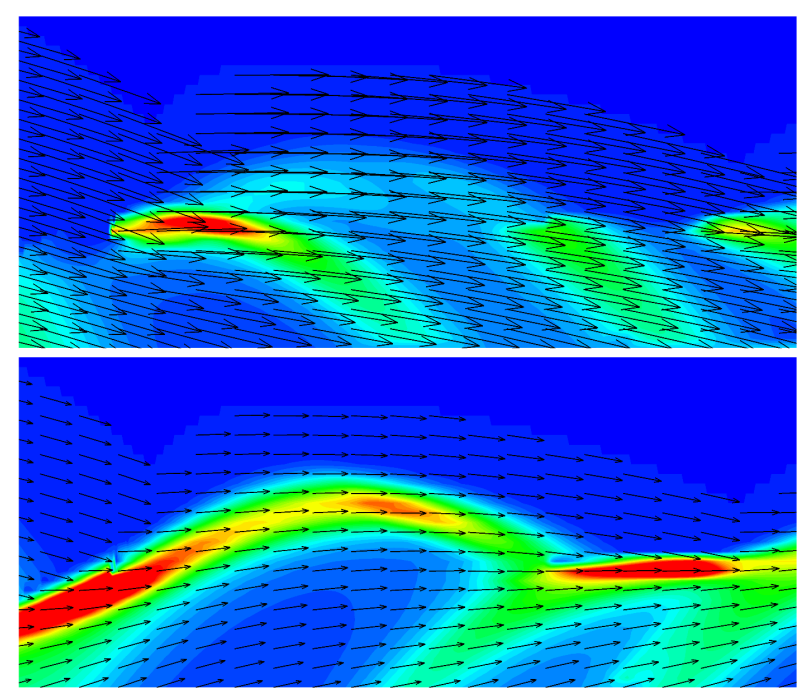

Figure 6 Particle distribution and particle velocity vectors in horizontal channel near the inlet (on the top is the concentration of all the particles $\mathrm{M}^{0}$, in the middle is the particle concentration and velocity vector distribution of node 1 , at the bottom is the particle concentration and velocity vector distribution of node 2)

From the bottom two figures, the particle concentration seen in the top $\mathrm{M}^{0}$ distribution are divided into two particle of the concentration which represent each node of the particles. Near the bottom, all the particle flow towards the wall in node1, and upwards off the surface in node2. This means that, at the wall, the injecting particles and the reflected particles are clearly and totally separated and represented by different particle flow fields. This is the reason why the VR-QMOM model can be used to simulate particle wall bouncing flows in the dilute particle concentration.

One benefit from the flow separation of the particle-wall collision, is that the pressure caused by this collision at the surface of the wall can be evaluated by the second order moment in the normal direction. In this simulation, the particle pressure at the surface is in proportion to the moment $\mathrm{M}_{\mathrm{jj}}^{2}$. Figure 7 shows the distribution of $\mathrm{M}_{\mathrm{jj}}^{2}$ at the bottom surface along the horizontal channel. The peaks show the high possibility of the pressure forcing on the local position. This prediction may give a reference value used for the pressure evaluation of the wall. 


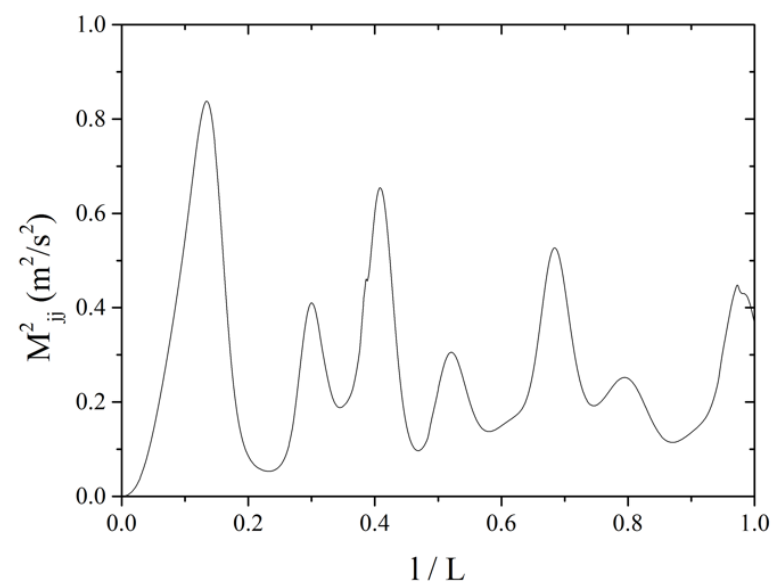

Figure 7. Second order moment of particles in the normal direction at the bottom surface along the channel.

\section{Conclusion}

The velocity-reconstructed two-node quadrature-based method of moment (VR-QMOM) has been presented and allows a more robust calculation of crossing and bouncing in the dilute multiphase simulation. The verification of the VR-QMOM model shows that it correctly predicts the crossing trajectories and the wall bouncing of particles. The two-node quadrature closure of VRQMOM requires the use of thirteen-moment equations for three-dimensional simulations. It makes the new VR-QMOM model much more computational efficient than the eight-node twenty-moment QMOM model whilst obtaining the same accuracy.

Using the Eulerian VR-QMOM method, the wall bouncing flow, which is a characteristic phenomenon of dilute particle flow, is successfully predicted. The simulation results from the VRQMOM Eulerian method have been compared with those from a Lagrangian method and agreement is very good. Coupling with the gas phase, the effect of the Stokes number is correctly predicted when varying the sizes of the particles and with VR-QMOM the competition of the gas carrier and the particle inertia in the particle-laden flow are correctly modelled. The distribution of the particle velocity and concentration at the surface shows the principle of the Eulerian model capable of prediction the dilute particles which is described by Lagrangian method. The evaluation of pressure 
on the surface by the parameters predicted directly from the simulation give an efficient engineering tools for the wear of the particle-laden pipelines.

\section{Acknowledgements}

This work has been funded by the Innovate UK (formerly the United Kingdom Technology Strategy Board, TSB) under SILOET II, in conjunction with Rolls-Royce PLC.

\section{References}

1 D. Miller. Pneumatic conveying design guide. (Second ed.), Oxford, 2004.

2 H. Kruggel-Emden, T. Oschmann, Numerical study of rope formation and dispersion of non-spherical particles during pneumatic conveying in a pipe bend, Powder Technology 268 (2014) 219-236.

3 C. Liang, John R. Grace, L. Shen, G. Yuan, X. Chen, C. Zhao, Experimental investigation of pressure letdown flow characteristics in dense-phase pneumatic conveying at high pressure, Powder Technology 277 (2015) 171-180.

4 F.T. Kus, M.A. Duchesne, S. Champagne, R.W. Hughes, D.Y. Lu, A. Macchi, P.Mehrani, Pressurized pneumatic conveying of pulverized fuels for entrained flow gasification, Powder Technology 287 (2016) 403-411.

5 David Mills. Pneumatic Conveying Design Guide. 2004

6 M. G. Jones, K. C. Williams, Predicting the mode of flow in pneumatic conveying systems-A review, Particuology 6 (2008) 289-300.

7 S. Lain, M. Sommerfeld, J. Kussin, Experimental studies and modelling of four-way coupling in particleladen horizontal channel flow, International Journal of Heat and Fluid Flow 23 (2002) 647-656.

8 M. Sommerfeld, J. Kussin, Wall roughness effects on pneumatic conveying of spherical particles in a narrow horizontal channel, Powder Technology 142 (2004) 180- 192. 
9 J. Kussin, M. Sommerfeld, Experimental studies on particle behaviour and turbulence modification in horizontal channel flow with different wall roughness, Experiments in Fluids 33 (2002) 143-159.

10 M. Sommerfeld, N. Huber, Experimental analysis and modelling of particle-wall collisions, International Journal of Multiphase Flow 25 (1999) 1457-1489.

11 N. Vásquez, K. Jacob, R. Cocco, S. Dhodapkar, G. E. Klinzing, Visual analysis of particle bouncing and its effect on pressure drop in dilute phase pneumatic conveying, Powder Technology 179 (2008) 170-175.

12 Y. Tsuji, Y. Morikawa, LDV measurements of an air-solid two-phase flow in a horizontal pipe, J. Fluid Mech. 120 (1982) 385-409..

13 Y. Tsuji, Y. Morikawa, LDV measurements of an air-solid two-phase flow in a vertical pipe, J. Fluid Mech. 139 (1984) 417-434.

14 Y. Tsuji, T. Oshima, Y. Morikawa, Numerical simulation of pneumatic conveying in a horizontal pipe, KONA 3 (1985) 38-51.

15 Y. Tsuji, Y. Morikawa, T. Tanaka, N. Nakatsukasa and M. Nakatani. Numerical simulation of gas-solid two-phase flow in a two-demensional horizontal channel, International Journal of Multiphase Flow 13 (1987) $671-684$.

16 Y. Tsuji, N. Y. Shen, Y. Morikawa, Lagrangian simulation of dilute gas-solid flows in a horizontal pipe, Advanced Powder Technology 2 (1991) 63-81.

17 M. Sommerfeld, Analysis of collision effects for turbulent gas-particle flow in a horizontal channel: Part I. Particle transport, International Journal of Multiphase Flow 29 (2003) 675-699.

18 M. Sommerfeld, J. Kussin, Analysis of collision effects for turbulent gas-particle flow in a horizontal channel. Part II. Integral properties and validation, International Journal of Multiphase Flow 29 (2003) 701718.

19 K. D. Squires, O. Simonin. LES-DPS of the effect of wall roughness on dispersed-phase transport in particle-laden turbulent channel flow. International Journal of Heat and Fluid Flow 27 (2006) 619-626. 
20 K. Li, S.B. Kuang, R.H. Pan, A.B. Yu, Numerical study of horizontal pneumatic conveying: Effect of material properties, Powder Technology 251 (2014) 15-24.

21 S. K. Pawar, J.T.Padding, N.G.Deen, A.Jongsma, F.Innings, J.A.M.Kuipers, Lagrangian modelling of dilute granular flow-modified stochastic DSMC versus deterministic DPM, Chemical Engineering Science. 105(2014) 132-142.

22 Qi. Zhou, L.S. Fan, A second-order accurate immersed boundary-lattice Boltzmann method for particleladen flows, Journal of Computational Physics 268(2014) 269-301.

23 S. A. Slater, J. B. Young, The calculation of inertial particle transport in dilute gas-particle flows, International Journal of Multiphase Flow 27 (2001) 61-87.

24 R.O. Fox, A quadrature-based third-order moment method for dilute gas-particle flows, Journal of Computational Physics 227 (2008) 6313-6350.

$25 \mathrm{H}$. Grad, On the kinetic theory of rarefied gases, Communications on Pure and Applied Mathematics 2 (1949) 331-407.

26 C. Lanczos, Applied Analysis, Dover Publications Inc., New York, 1988.

27 O. Desjardins, R.O. Fox, P. Villedieu, A quadrature-based moment method for dilute fluid-particle flows, Journal of Computational Physics 227 (2008) 2514-2539.

$28 \mathrm{H}$. Grad, On the kinetic theory of rarefied gases, Communications on Pure and Applied Mathematics 2 (1949) 331-407.

29 L. Schiller, A. Nauman, A drag coefficient correlation, V.D.I. Zeitung 77 (1935) $318-320$.

30 C. Yoon, R. McGraw, Representation of generally mixed multivariate aerosols by the quadrature method of moments: I. Statistical foundation, Aerosol Science 35 (2004) 561-576.

31 V. Vikas, Z. J. Wang, A. Passalacqua, R.O. Fox, Realizable high-order finite-volume schemes for quadrature-based moment methods, Journal of Computational Physics 230 (2011) 5328-5352. 
32 P. L. Roe, Characteristic-based schemes for the Euler equations, Ann. Rev. Fluid Mech. 18(1986) 337365.

33 S. Laín, M. Sommerfeld. Euler/Lagrange computations of pneumatic conveying in a horizontal channel with different wall roughness. Powder Technology. 184 (2008) 76 - 88 